\title{
A Rare Cause of Abdominal Pain: Mucocele of Appendix
}

\author{
Mountassir Moujahid1', Issam Ennafaa², El Ghari Mohamed², Issam Serghini³, \\ Moulay El Hassan Tahiri ${ }^{1}$ \\ ${ }^{1}$ Department of General Surgery, 5th Miltary Hospital, Guelmim, Morocco \\ ${ }^{2}$ Department of Radiology, 5th Miltary Hospital, Guelmim, Morocco \\ ${ }^{3}$ Department of Reanimation, 5th Miltary Hospital, Guelmim, Morocco \\ Email: ${ }^{*}$ m.moujahid@gmx.fr
}

Received 8 January 2014; revised 4 February 2014; accepted 3 March 2014

Copyright (C) 2014 by authors and Scientific Research Publishing Inc.

This work is licensed under the Creative Commons Attribution International License (CC BY).

http://creativecommons.org/licenses/by/4.0/

(c) (i) Open Access

\begin{abstract}
The mucocele of appendix or mucosecreting tumor is a rare affection defined as a cystic dilatation of the light of the appendix following the intra luminal accumulation of mucinous, translucent, gelatinous, secretion which may reach either the entire organ or a segment more often distal. The pathogenesisis is discussed; the diagnosis is more often preoperative. The therapeutic depends on the histological nature. This goes from the simple appendicectomy, whose shapes are benign to the right hemicolectomy in the malignant mucocele. We report a case collaged in the general surgical service of the $5^{\text {th }}$ Military Hospital.
\end{abstract}

\section{Keywords}

Appendix; Mucocele; Peritoneal Pseudomyxoma; Diagnosis; Surgical Treatment

\section{Introduction}

The appendicular mucocele is a dilation of the appendix by accumulation of secondary mucus in an obstruction of its light which can be of multiple origins. It is a rare pathology. The clinical symptom is very varied [1]. The diagnosis is often evoked in the imaging and confirmed by the anatomo pathologic study allowing an adapted surgical treatment.

We report a case collaged in the service. Through a review of the literature, we try to clear the various diagnostic ways and the anatomo pathological aspects from which ensues the surgical treatment.

${ }^{*}$ Corresponding author. 


\section{Observation}

A 60-year-old man without notable pathological histories, consulted in emergencies for pains of the right iliac pit evolving for 15 days. There was no fever but a sensibility of the right iliac pit and no notion of nausea or vomiting. The biological balance showed a hyperleukocytosis without inflammatory syndrom. The clinical examination noted a pain provoked in the palpation of the right iliac pit and the presence of a mass fixes to the plan $5 \mathrm{~cm}$ deep of main line. The abdominal-pelvic spiral scanning was realized with injection of contrast in axial cups (Figure 1), and sagittal reconstructions (Figure 2) showed the presence of a cystic mass of the right iliac pit in juxta caecal elongated, whose wall raised after injection of contrast. The diagnosis of mucocele of the appendix was put. The patient was operated as a matter of urgency by a median laparotomy with realization of a right hemi colectomy and a terminal ileo colic anastomosis (Figure 3). The anatomopathologic report that there were at the level of the internal face of the cyst a fibrin deposit and mucoid material compatibly with a mucocele of the appendix.

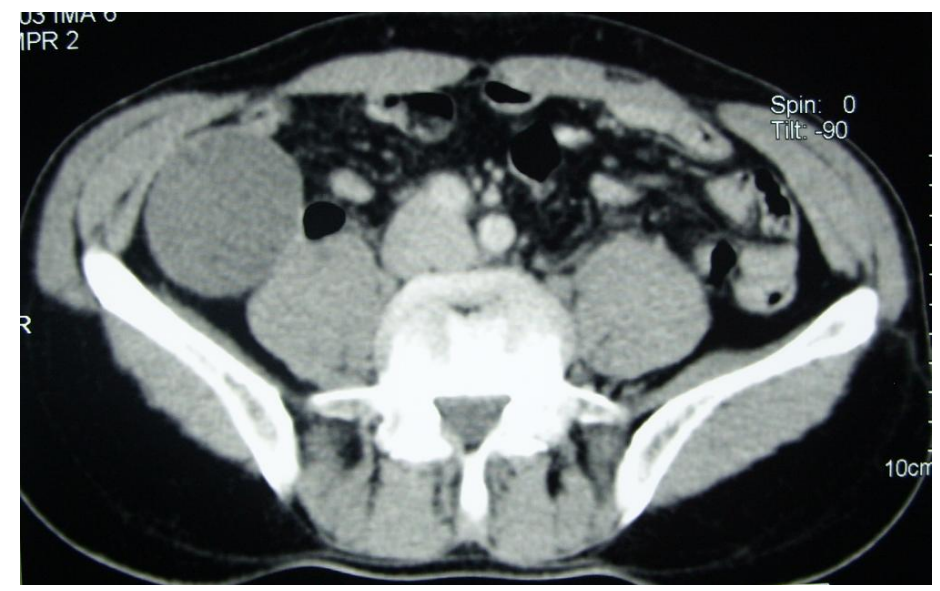

Figure 1. Abdominal pelvic scan showing a cystic formation of the right iliac pit which wall raises after injection.

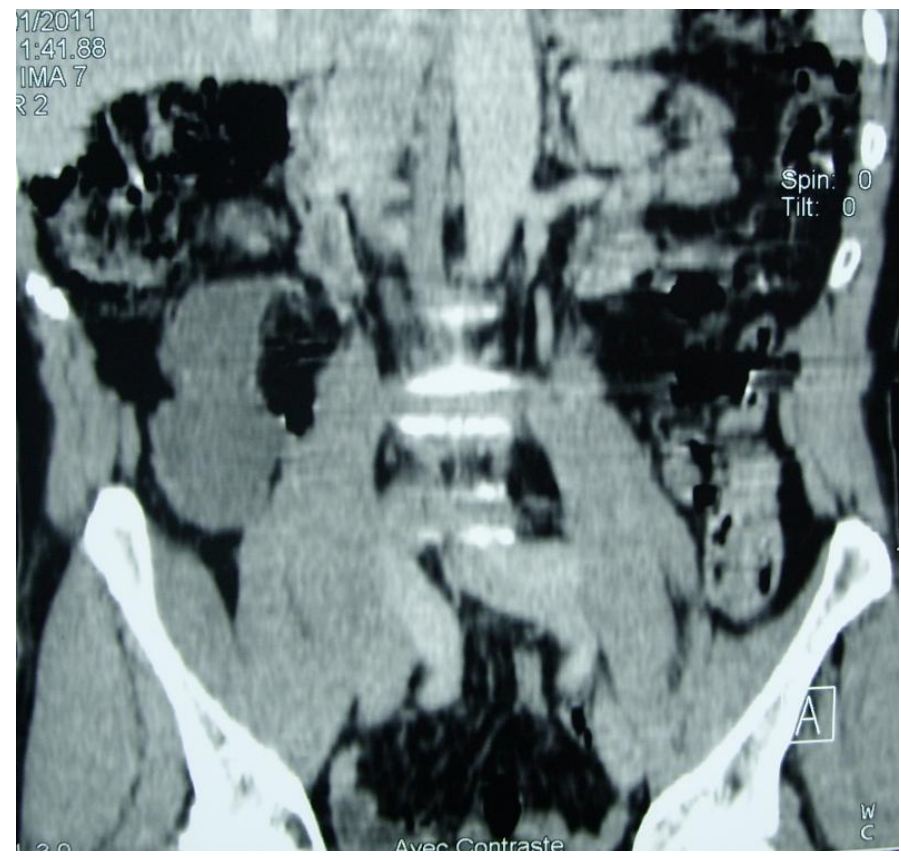

Figure 2. Abdominal pelvic scan in sagittal reconstructions showing the elongated character of the cystic in juxta caecum formation. 


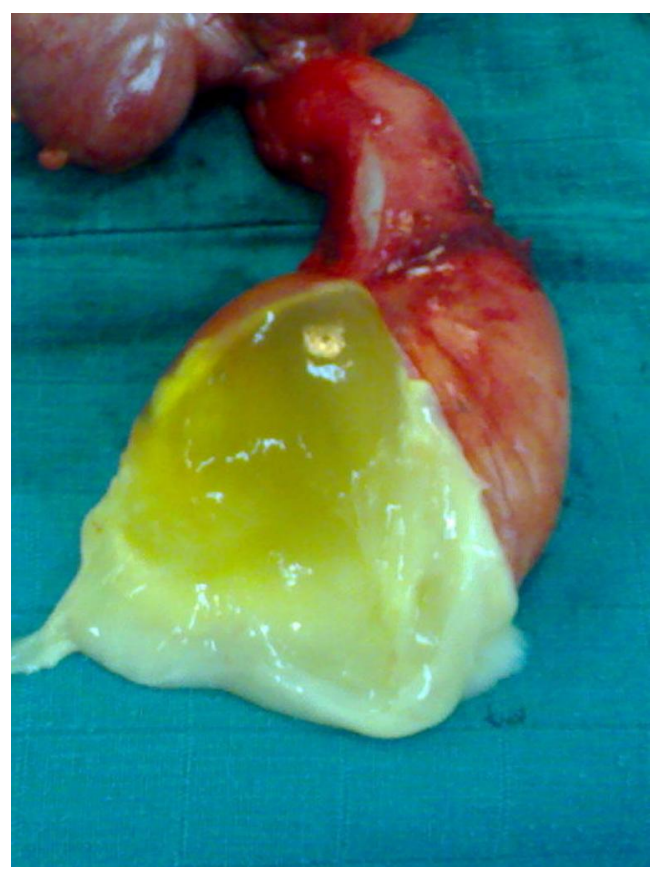

Figure 3. Macroscopic view after opening of the appendix with gelatinous contents.

The follow up were simple without any sign of recurrence.

\section{Discussion}

The mucocele of appendix is a rare pathology estimated at $0.2 \%$ and $0.3 \%$ of appendicectomy [1]. It corresponds to a dilation of the appendix by accumulation of secondary mucus in an obstruction of the light either of mechanical origin in case of ligature or of stercolithe, or by loco regional achievement in case of benign tumors: endometriosis, adenoma villeux or malignant adenoma, carcinoma, carcinoïd tumor [2].

The term of mucocele appendix is introduced into the medical language only in 1876 by FERE [3]. It affects preferentially the woman between 50 and 60 years [1].

The clinical symptom is variable; the rarity of the affection and the absence of the specificity of the symptom make that the diagnosis was almost never put in preoperative. In $23 \%$ of the cases, mucocele of appendix is asymptomatic.

In most of the cases it is about chronic pains of the right iliac pit (48\% - 64\% of the cases]. These pains can be occasional or feign a board of acute appendicitis. In 18 at 32\% of the cases the mucocele shows itself by a tangible mass of the right iliac pit [4] as it was the case at our patient. More rarely, the mucocele shows itself by an acute intestinal obstruction [2], a low digestive bleeding [1] or a peritonitis [3].

Because of the diagnostic difficulty and the little suggestive circumstances of discovery, the complementary examinations, in particular the imaging (the echograph and the spiral scanning), find a major place [1] [3].

In the echograph it appears as an elongated mass of the right iliac pit hypo echogen with fine echo parietal calcifications [1] [3] [4].

The scanner is the most successful exam. It showed hypodense mass the calcified wall which rises after injection of contrast. It allows to look for signs of peritoneal scattering with presence of a dense ascite or peritoneal nodules [1] [2].

The biological balance brings no help for the diagnosis of the mucocele appendix.

The main differential diagnoses are the cysts of the mesentery, the digestive duplications, the complicated appendicitis, the ovarian cysts and the hydrosalpinx [2].

The mucocele of appendix can complicate a gelatinous disease of the peritoneum in 5 at $15 \%$ of the cases [2] [5]. The perforing made in the peritoneal cavity in most of the cases, this gelatinous effusion is called pseudomyxoma peritonei. The recurrence is frequent. The appendicular origin represents $30 \%$ of the cases of the gela- 
tinous diseases [5].

The treatment and the prognosis depend on the histology. So, the mild forms benefit from an appendicectomy which can be made by a Mac Burney or a laparoscopic way [2], sometimes combined in a resection of the low cæcal bottom. The mild forms are cured by the surgery and do not do it again after complete resection with a rate of survival in 5 years which varies from $85 \%$ to $100 \%$ [5].

In case of malignity right hemi colectomy is justified and the survival in 5 years vary $25 \%$ to $38 \%$ according to the series [3] [5].

It is necessary to note that the intervention realized by a laparotomy or by a laparoscopy, a protection of the operative field, a good peritoneal toilet especially in case of break and the extraction in the endobag, the precautions must be taken.

On the histological plan mucocele is divided into three according to the criteria of the WHO: simple mucocele, cystadenoma and cystadenocarcinoma [4].

The therapeutic attitude in case of break of the appendicular mucocele is however dark. This break is often associated with a mucineux adenocarcinoma and thus with a metastatic intra peritoneum scattering.

It seems that it is necessary to remove a maximum of mucine and to resect the appendix, the right colon and the epiploon.

The rinsing of the abdominal cavity by a mucolytic agent can prevent the recurrence. The adjuvant intra peritoneum chemotherapy by 5 fluoro-uracil, thiotepa, melphalan; cisplatine, mitomycine $\mathrm{C}$ and chlorambucil is well tolerated but their effect is fickle [2] [5].

The systematic chemotherapy, the radiotherapy and the immunotherapy are debated [1]-[4].

Finally, we can quote the interest of the systematic hyperthermia mainly in case of pseudomyxoma peritonei [5].

\section{Conclusion}

The mucocele of appendix is a rare pathology of varied symptom which must be evoked in front of any cystic mass of the right iliac pit. The imaging and the histology allow confirming the diagnosis and specifying the degree of wickedness. The surgical treatment must be early to avoid the peritoneal break.

\section{References}

[1] Weber, G., Teriitehau, C., Goudard, Y., et al. (2009) Mucocele of Appendix. Feuillets of Radiology, 49, 40-44. http://dx.doi.org/10.1016/S0181-9801(09)71565-9

[2] Armstrong, O., Lepourry, J., Frampas, E., et al. (2010) Mucocele of Appendix: Interest of a Coelio Conversion of Caution and a Follow-Up. Gastroenterology Clinical and Biological, 34, 416-419.

[3] Kim, S., Lim, J.H., Lee, W., et al. (1998) Mucocele of the Appendix: Ultrasonographic and CT Findings. Abdomen Imaging, 23, 292-296. http://dx.doi.org/10.1007/s002619900343

[4] Roberge, R.J. and Park, A.J. (2006) Mucocele of the Appendix: An Important Clinical Rarity. The Journal of Emergency Medicine, 30, 303-306. http://dx.doi.org/10.1016/j.jemermed.2005.05.026

[5] Fairise, A., Barbary, C., Derelle, A.L., et al. (2008) Mucocele of Appendix and Pseudomyxoma Péritonei. Journal de Radiologie, 89, 751-762. http://dx.doi.org/10.1016/S0221-0363(08)73781-8 